\title{
Cuestiones institucionales en el Acuerdo de 1994 relativo a ia parte XI de la Convención sobre el Derecho deī Mar
}

\author{
Felipe H. Paolillo
}

\begin{abstract}
El Acuerdo de julio de 1994 introdujo profundos cambios en las normas de la Parte XI de la Convención sobre el Derecho del Mar que regulan los procedimientos para la adopción de decisiones en la Ásamblea y el Consejo, la composición de este último y la distribución de competencias entre los dos órganos. Como resultado de dichos cambios se acentúa la supremacía que de hecho tenía el Consejo sobre la Asamblea (sólo formalmente "el órgano supremo" en el sistema de la Convención) y se fortalece la posición dentro del primero de los países industrializados, que se encuentran ahora con posibilidad de poder controlar y orientar la gestión de la Autoridad.
\end{abstract}

\section{I}

Los esfuerzos iniciados en 1990 por el ex-Secretario General de las Naciones Unidas, Sr. Javier Pérez de Cuéllar, para lograr el más amplio apoyo de los Estados a la Convención de las Naciones Unidas sobre el Derecho del Mar de 1982 (en adelante: "la Convención"), culminaron exitosamente el 28 de julio de 1994, cuando la Asamblea General aprobó la resolución 48/263 que contiene el "Acuerdo relativo a la aplicación de la Parte XI de la Convención" (en adelante: "el Ảcuerdo"). ${ }^{\text {? }}$

Las negociaciones que precedieron la adopción del Acuerdo consistieron en consultas informales que se desarrollaron bajo la dirección del propio ex-Secretario General, y luego bajo la de su sucesor Sr. Boutros

\footnotetext{
${ }^{1}$ Aprobada por 121 votos, ninguno en contra y 7 abstenciones.
} 
Boutros-Ghali, y en la que participaron alrededor de 80 países. El propósito de dichas negociaciones fue el de encontrar fórmulas para satisfacer los intereses de los países industrializados que habían objetado severamente el sistema de administración y explotación de la zona internacional de los fondos marinos (en adelante "la Zona") tal como había sido consagrado en la Parte XI de la Convención.

La certeza de que los Estados que, precisamente, poseían la capacidad para emprender actividades en la Zona no ratificarían la Convención en tanto se mantuviera el sistema previsto en la Parte XI, fue lo que creó la necesidad de buscar formas de superar las objeciones a los efectos de promover la aceptación universal de la Convención. Esta necesidad se agudizó cuando el 16 de noviembre de 1993 se depositó el sexagésimo instrumento de ratificación de la Convención, lo cual determinó que 12 meses más tarde la misma entrara en vigor. Ello ocurriría, sin embargo, casi exclusivamente entre países en desarrollo. ${ }^{2}$ Tanto para los Estados partes de la Convención como para los que se mantenían fuera de ella, su entrada en vigor sólo para un tercio de los miembros de la comunidad internacional, ninguno de los cuales pertenecía el grupo de los países industrializados más importantes, constituía un hecho poco auspicioso que no permitía predecir que algún día ella alcanzaría una vigencia efectiva y universal.

Si por un lado la necesidad de atraer a la órbita de la Convención al grupo de paises industrializados justificó la realización de consultas y negociaciones encaminadas a introducir ajustes en la Parte.XI, por otro lado los cambios ocurridos en la última década en el contexto político internacional facilitaron dicha tarea. Dichos cambios tuvieron lugar bajo el signo de la declinación de ciertas ideologías sobre las que se habían fundado la posiciones adoptadas por algunos grupos de Estados en la Tercera Conferencia de las Naciones Unidas sobre el Derecho del Mar (en adelante: "la Conferencia") en relación con el régimen legal de la Zona y sus recursos, y de un paralelo resurgimiento generalizado de los principios de la economía de mercado. Ello condujo a la emergencia de un panorama internacional muy diferente al que prevaleció durante el período de gestación de la Convención. La Asamblea General, en uno de los párrafos preambulares de su resolución 48/263, invoca estos cambios para justificar "la reevaluación de algunos aspectos del régimen relativo a la Zona y sus recursos".

${ }^{2}$ Todos los paises que habian ratificado la Convención hasta ese momento pertenecian al grupo de paises en desarrollo, excepto Islandia. 
Por otra parte, la necesidad de iniciar las actividades de explotación de los recursos de la Zona, que se había presentado al comenzar la Conferencia como una alternativa inaplazable, se fue diluyendo como consecuencia del deterioro del mercado mundial de metales; actualmente se considera que la iniciación de las actividades de explotación de los recursos minerales de los fondos marinos no tendrá lugar sino después que entremos en el próximo siglo.

El Acuerdo contiene en un "Anexo" las substanciales enmiendas que se introdujeron a la Parte XI mediante un mecanismo de modificación que la Convención no prevé. En ninguna parte de la resolución, del Acuerdo o del Anexo se alude a "enmiendas" o "cambios". Lo que se dice en dichos textos es que ciertas disposiciones de la Parte XI o del Anexo III a la Convención no serán aplicadas, o que la Parte XI se aplicará de conformidad con el Acuerdo (art. 1 del Acuerdo), o que las disposiciones de la Parte XI y del Acuerdo deberán ser interpretadas y aplicadas en forma conjunta como un solo instrumento (cuarto párrafo dispositivo de la resolución; art. 2, par. 1 del Acuerdo). Estas expresiones no quieren decir necesariamente lo mismo. De todos modos, lo que és claro es que toda vez que la Convención y el Acuerdo prescriban cósas diferentes, deben aplicarse las disposiciones del segundo, quedando los correspondientes artículos de la primera sin valor legal alguno (art. 2 , par. 1 del Acuerdo).

A pesar de que el Acuerdo establece procedimientos muy flexibles para facilitar su rápida entrada en vigor (arts. 4 y 5), se estimó que séría muy difícil que tódos los Estados que habían ratificado la Convención liegaran a ser partes de aquel antes del 16 de noviembre de 1994. Es por ello que en dicho instrumento se incluyeron disposiciones que permiten su aplicación provisional de manera de asegurár que al entrar en vigor de la Convención, su Parte XI y el Acuérdo puedan interpretarse y aplicarse en forma conjunta. Hasta el momento de escribirse esta nota (abril de 1995), 105 Estados han manifestado que aplicarán el Acuerdo provisionalmente, de los cuales 37 han ratificado la Convención. El número total de Estados que han ratificado la Convención hasta el momento es de 72, entre los que figuran algunos miembros del grupo de países desarrollados, ${ }^{3}$ grupo que había estado conspicuamente ausente de la lista de Estados Partes hasta la adopción del Acuerdo de junio de 1994.

\footnotetext{
${ }^{3}$ Ellos son: Alemania, Australia e Italia, todos los cuales depositaron sus instrumentos de ratificación 0 adhesión con posterioridad a la adopción de la resol. 48/2636.
} 
Es previsible que el ritmo de ratificaciones y adhesiones se acelere en el futuro inmediato, y se espera que no habrá que esperar mucho para que la Convención haya adquirido la vigencia universal o casi universal que se buscó con la celebración del Acuerdo. Mientras tanto, en virtud de los mecanismos previstos en el Acuerdo relativos a su vigencia y aplicación provisional (arts. 4 a 8); la Autoridad Internacional de los Fondos Marinos (en adelante: "la Autoridad") estará integrada por dos clases de miembros: los Estados que son partes de la Convención y aquellos que no son partes pero que aceptaron aplicar provisionalmente el Acuerdo. Estos últimos son miembros provisionales de la Autoridad con los mismos derechos y obligaciones que los miembros natos (Acuerdo, Anexo, Sección 1, pár. 12). ${ }^{4}$

\section{II}

El Acuerdo contiene nuevas reglas que rigen las numerosas cuestiones cuya regulación en la Convención presentaba dificultades para los países industrializados y que son las siguientes: lö costos para los Estados que resultan del fücionamiento de la Autoridad; lós arreglos institúciónales relacionados con la conducción de las actividades en la Zona; la Empresa; la adopción de decisiones por parte de los órganós de la Autoridad; la Conferencia de Revisión; la transferencia de tecnología; la política de producción; la asistencia económica; las disposiciones financieras de los contratos y el medio ambiente marino. 5

Tanto los cambios introducidos en el sistema de administración y explotación de la Zona como los aspectos formales relativós ala entrada en vigor del Acuerdo y a la compleja relación entre éstè y la Convención, plantean interesantes problemas jurídicos que no serán objeto de análisis en esta nota. Aquí nos referiremos solamente a uno de los aspectos del funcionamiento de la Autoridad que ha sido regulado en el Acuerdo de un modo muy diferente al que se preveía en la Convención: el sistema de adopción de decisiones de los órganos de la Autoridad.

\footnotetext{
${ }^{4}$ Cfr. Moritaka Hayashi, "UNCLOS's Part XI Agreement, with Particular Reference to the Status of Registered Pioneer Investors", trabajo presentado en la International Conference on Deep Seabed Mining Policy, Seúl, Septiembre 1994, p. 11.

${ }^{5}$ A ciertá altura de las consultas se reconoció que aun cuando las consideraciones ambientales eran de la mayor importancia en el contexto del Derecho del Mar, ellas no llegaban a constituir un obstáculo para lograr la aceptación universal de la Convención. En consecuencia se resolvió dejar la discusión de este tema para una furtura instancia. Cfr. D.H. Anderson, "Efforts to Ensure Universal Participation in the United Nations Convention on the Law of the Sea", International and Comparative Law Quarterly, Vol. 42, Part 3, July 1993, p. 660).
} 


\section{III}

\section{Organos y funciones de la Autoridad de acuerdo con la Con- vención.}

A primera vista la estructura orgánica de la Autoridad, tal como ha sido prevista en la Convención (Parte XI, Sección 4) sigue el modelo tradicional adoptado por la mayor parte de las organizaciones internacionales, que consiste en el establecimiento de un órgano pierario (la Asamblea), de un órgano de composición restringida (el Consejo) y de una Secretaría. ${ }^{6}$ Estos son los órganos "principales" de la Autoridad, a los que se agrega un cuarto órgano operativo, con características originales, llamado la Empresa (art. 158, párrafos 1 y 2). La Asamblea, el órgano "supremo" (art. 160, par. 1) está integrada por todos los miembros de la Autoridad, o sea, por todos los Estados y otras entidades que de acuerdo al art. 305 pueden ser partes de la Convención. ${ }^{7}$ El Consejo es el órgano ejecutivo y se integra con 36 miembros elegidos por la Asamblea de acuerdo con reglas que se examinan infra.

No existe en la Convención un criterio general de distribución de competencias entre estos dos órganos principales. La regla contenida en el artículo 158, par. 4 sobre cómo los órganos principales de la Autoridad y la Empresa deben ejercer sus facultades y funciones es superflua y no sirve para resolver el problema que se presenta en los casos de concurrencia de competencia o en los casos de poderes no específicamente asignados a ninguno de los dos órganos. (La Parte XI y los anexos a la Convención, en particular el Anexo III, atribuyen funciones a la Autoridad sin especificar, en algunos casos, a qué órgano corresponde desempeñarlas).

De la lectura de los arts. 160 (Facultades y funciones de la Asamblea) y 162 (Facultades y funciones del Consejo), se llega a la previsible conclusión de que la Asamblea es el órgano deliberante de la Autoridad y el Consejo el órgano ejecutivo. Pero a la correspondiente característica

\footnotetext{
${ }^{6}$ Para un análisis detallado de la organización y funciones de la Autoridad ver Felipe H. Paolillo, "The Institutional Arrangements for the Intemational Sea-Bed and Their Impact on the Evolution of International Organizations", Recueil des Cours de l'Academie de Droit International, Vol. 188, p. 139.

${ }^{7}$ Dichas entidades pueden ser Estados asociados autónomos [pár. I, incisos c) y d)]; territorios que gocen de plena autonomía interna pero que no hayan alcanzado la plena independencia [inc. e] y organizaciones internacionales [inc. f].
} 
de cada órgano debiera agregarse la calificación "predominantemente", porque ni la primera es exclusivamente deliberante ni el segundo es exclusivamente ejecutivo. El ejercicio de las principales funciones políticas y normativas (adopción de políticas de la Autoridad; adopción de reglas, reglamentos y procedimientos) es compartida por los dos órganos, del mismo modo que lo es el ejercicio de algunas funciones administrativas y ejecutivas (elección de autoridades; aprobación del presupuesto, adopción de medidas de ajuste económico, suspensión en el ejercicio de derechos y privilegios de los miembros).

El sistema de votación en la Asamblea es, en principio, similar al de los órganos plenarios de otras instituciones internacionales, es decir, mayoría simple de miembros presentes y votantes para decidir cuestiones de procedimiento; y mayoría de dos tercios de miembros presentes y votantes para las cuestiones de fondo, siempre que dicha mayoría comprenda la mayoría de los miembros que participen en el período de sesiones. (art. 159, párs. 7 y 8 ).

Sólo en un caso se requiere el consenso de la Asamblea: cuando las contribuciones de los Estados Partes para financiar la operación inicial de la Empresa son insuficientes, la Asamblea decidirá por consenso qué medidas adoptar para hacer frente al déficit [Anexo IV, art. 11, 3, (c)]. El art. 159 prevé, además, dos procedimientos especiales relativos a la adopción de decisiones: uno, para aplazar la decisión de someter a votación una cuestión de fondo (par. 9); el otro para pedir que la Sala de Controversias de los Fondos Marinos del Tribunal Internacional del Derecho del Mar emita una opinión consultiva acerca de la conformidad con la Convención de una propuesta sometida a la Asamblea (par. 10).

El sistema de votación del Consejo, en cambio, es extraordinariamente complicado. Prácticamente todos los procedimientos de voto concebibles fueron considerados y discutidos durante las negociaciones en la Conferencia, entre otros: mayoría simple, de dos tercios, de dos tercios más uno, de tres cuartos, minorías de $2,3,5,7$ ó 9 miembros con capacidad de veto (blocking minorities), voto ponderado, veto, mayorías concurrentes, votos regionales, mayorías de grupos de interés y consenso. Es que siendo el Consejo el órgano que adoptará las decisiones más importantes, el sistema de votación en dicho órgano se convirtió en uno de los temas más controvertidos de la Conferencia. Cada grupo de Estados con intereses comunes buscó imponer el sistema que les asegurara un perfecto control sobre las actividades del órgano de modo de asegurar que ninguna decisión fuera adoptada contra su voluntad, garantizando de este modo la protección de dichos intereses. 


\section{F. H. Paolillo / Cuestiones institucionales en el ...}

Después de prolongadísimas y complicadas negociaciones se llegó al resultado que se refleja en el art. 161, par. 8 de la Convención, que contiene lo que tal vez sea el sistema de votación más intrincado que se haya concebido para una organización internacional. Las cuestiones que el Consejo debe resolver se dividen en 5 categorías, y para cada una de ellas se establecen distintas mayorías: cuestiones de procedimiento que se resuelven por simple mayoría de miembros presentes y votantes; ${ }^{8}$ cuestiones de fondo que se deciden por una mayoría de dos tercios de miembros presentes y votantes siempre que dicha mayoría incluya la mayoría de los miembros del Consejo; cuestiones de fondo para cuya decisión se requiere una mayoría de tres cuartos de los miembros presentes y votantes que incluya la mayoría de los miembros del Consejo; cuestiones que requieren consenso, y por último las decisiones relativas a los planes de trabajo que presentan los Estados, la Empresa y las otras entidades a las que se refiere el art. 153, par. 2, para tener acceso a los recursos de la Zona y proceder a su explotación, y que están sometidas a un procedimiento especial.

Para algunos países industrializados el sistema establecido en la Convención de representación y de votación en el Consejo, conjuntamente con la forma en que las competencias han sido distribuidas entre los dos órganos principales de la Autoridad, no garantizaban suficientemente la protección de sus intereses. Dichos países entendían que, siendo los que mayores riesgos y responsabilidades asumirian en la explotación de los recursos de la Zona, y por lo tanto los que serían más afectados por las decisiones del Consejo, la Convención no les acordaba un poder de influencia dentro de dicho órgano proporcionado a sus intereses. Por tal razón, la cuestión de la adopción de las decisiones por parte de los órganos de la Autoridad fue uno de los nueve temas que fueron objeto de negociación durante las consultas informales 1990-1994.

El sistema que emergió de dichas consultas y que se describe en los 16 párrafos de la Sección 3 del Acuerdo, introduce modificaciones substanciales en la Parte XI de la Convención, no sólo en lo que se refiere a los procedimientos de adopción de las decisiones de la Asamblea y el Consejo, sino también en lo que respecta a la composición del Conisejo y a la distribución de competencias entre ambos órganos.

\footnotetext{
${ }^{8}$ Debe notarse que ciertas cuestiones tradicionalmente consideradas como cuestiones de procedimiento son consideradas cuestiones de substancia en la Convención, como por ejemplo la solicitud de opiniones consultivas (art. 191) o la creación de órganos subsidiarios, [art. 162, par. 2 (d)].
} 


\section{IV}

\section{Cambios en la distribución de competencias.}

De acuerdo con la Convención es a ia Asamblea a la que corresponde "establecer...Ja política genieral de la Autoridad respecto de todas las cuestiones de la competencia de ésta" (art. 160, par. 1), en tanto que el Consejo se limita a establecer la política "concreta" que seguirá la Autoridad en relación con toda cuestión o asunto de su competencia "de conformidad con esta Convención y con la política general establecida por la Asamblea" (art." 162; par, 1). ${ }^{9}$ Es clara, entonces, la situación de subordinación en que la Convención coloca al Consejo con relación a la Asamblea en lo que a la determinación de la política đe la Autoridad se refiere, no sólo porque aquél no interviene en la formulación de la política "general" sino porque, además, al formular la política "concreta" el Consejo debe ajustarse a la política general dictada por la Asamblea.

Esta es, en realidad, la única manifestación del carácter de órgano "supremo" que la Convención le atribuye a la Asamblea (art. 160, par. 1), ya que en todos los demás aspectos es el Consejo el órgano en el que se concentran los mayores poderes lo cual lo convierte en el órgano efectivamente supremo dentro de la Autoridad. ${ }^{10}$

La calificación de "suprema" le fue acordada a la Asamblea en razón de la insistencia de los países del Grupo de los 77 que entendían que al órgano con mayor carácter representativo (y en el cual, por lo tanto, el Grupo constituía la mayoría), debía acordársele una superioridad jerárquica. A diferencia de los primeros textos de negociación, el texto de la Convención vincula el carácter supremo de la Asamblea al hecho de ser el "único órgano integrado por todos los miembros de la Autoridad", revelando de este modo que no se trata de una supremacía jurídica resultante de atribuírsele mayores funciones y poderes, sino de una supremacia formal derivada de su carácter representativo. Esta fue la interpretación de los países industrializados, y también la del Presidente de la Primera Comisión de la Conferencia en donde se negoció el texto,

\footnotetext{
${ }^{9}$ No es fácil establecer el límite que separa la política general de la política concreta de la Autoridad. ¿Dónde termina una y empieza la otra? Ni del texto de la Convención ni de sus antecedentes es posible inferir un criterio que permita distinguir una política de la otra. El problema desaparece prácticamente con la entrada en vigor del Acuerdo, dado que el Consejo pasa a desempeñar un papel preeminente en la elaboración de las dos.

${ }^{10}$ Paolillo, op. cit., p. 244.
} 
quien expresó que la palabra "supremo" en el art. 160, par. 1 había sìto. empleada "solamente para describir el órgano más representativo de los" miembros de la humanidad". 11

En los hechos, el carácter supremo de un órgano resulta menos del lenguaje que se usó para definirlo que de la naturaleza y númèro de funciones que se le asignen y de los poderes que se le atribuyan para desempeñarlas. En el caso de la Autoridad, un cuidadoso examen comparativo de las funciones y poderes de la Asamblea y del Consejo lleva a la inequívoca conclusión de que es en el Consejo en donde se concentran los mayores poderes de la Autoridad. El Consejo supervisa la aplicación de las disposiciones de la Parte XI de la Convención, tiene a su cargo la administración de la Zona, decide cómo y cuándo se realizará la explotación de sus recursos y participa, en algunos casos en forma preeminente, en la formulación de la políticas y en la actividad legislativa y reglamentaria de la Autoridad. 12

Aun así, para algunos países industrializados resultaba inaceptable el hecho de que la formulación de la política general de la Autoridad fuera decidida por un órgano de carácter plenario en donde la superioridad numérica de los países en desarrollo y el sistema de votación no les permitía ejercer un efectivo control sobre las decisiones. Por ello las negociaciones que llevaron a la adopción del Acuerdo fueron orientadas a disminuir la participación que la Convención había asignado a la Asamblea en la formulación de la política de la Autoridad. El resultado fue una verdadera inversión de los papeles, ya que de conformidad con el Acuerdo, la Asamblea queda ahora subordinada al Consejo.

El primer párrafo de la Sección 3 del Acuerdo dispone que "La Asamblea, en colaboración con el Consejo, determinará la política general de la Autoridad". Esta disposición cambia completamente la relación existente entre los dos órganos en esta materia ya que la política general de la Autoridad, materia de exclusiva competencia de la Asamblea de acuerdo con la Convención, se convierte, por virtud del Acuerdo, en un asunto respecto del cual el Consejo tambiéri tiene competencia $\mathrm{Y}$ de conformidad con el párrafo 4 de la Sección 3 del Acuerdo, en cuestiones de competencia compartida la Asamblea debe adoptar sus decisiones basándose en las recomendaciones del Consejo. Si la Asamblea no acepta las recomendaciones del Consejo, el asunto es devuelto para que éste lo examine teniendo presentes las opiniones expresadas por la Asamblea.

11 Official Records of the Conference, vol. V, p. 127.

${ }^{12}$ Paolillo, loc. cit, p. 242. 
Al perder la íniciativa y la posibilidad de adoptar en forma autónoma la política general de la Autoridad, la Asamblea ha perdido una de las pocas funciones de substancia que la Convención le había atribuido de manera exclusiva.

El Acuerdo cercena los poderes de la Asamblea en otra área que, de conformidad con la Convención, era de su exclusiva competencia. En efecto, la determinación de las cuotas de los miembros en el presupuesto de la Autoridad es materia que le corresponde a la Asamblea según el art. 160, par. $2 ;$ e) de la Convención. El Acuerdo dispone que los órganos competentes para entender-en cuestiones que tengan consecuencias financieras o presupuestarias son la Asamblea y el Consejo, sin especificar qué corresponde a quién, y agrega que las decisiones que se adopten en relación con tales cuestiones deberán estar basadas en las recomendaciones del Comité de Finanzas, órgano que se crea por el mismo Acuerdo y que probablemente funcionará como órgano subsidiario del Consejo (ver infra).

\section{V}

\section{Cambios en el sistema de votación.}

i) El consenso como principio general: El Acuerdo introduce varios cambios substanciales en lo que se refiere a los procedimientos de adopción de decisiones. El párrafo 2 de la Sección 3 formula un principio general que se aplica a todas las decisiones de los órganos de la Autoridad y que no tiene precedentes en la Convención:

"Como norma general, las decisiones de los órganos de la Autoridad se deberán adoptar por consenso."

En la Convención se requiere el consenso de la Asamblea sólo para un asunto [medidas para hacer frente al déficit de los fondos que deben proporcionarse a la Empresa, Anexo IV, art. 11,3 (c) ${ }^{13}$ En cambio el Consejo debe decidir por consenso varias cuestiones de fondo, algunas de las cuales aparecen citadas en el art. 161, par. 8, d) (medidas para la

\footnotetext{
${ }^{13}$ Las Reglas de Procedimiento de la Comisiön Preparatoria de la Autoridad de los Fondos Marinos y del Tribunal Internacional del Derecho del Mar incluyeron la cuestión de la determinación de las cuotas de los miembros en el presupuesto administrativo de la Autoridad entre las que requieren el consenso de la Asamblea (art. 160, par. 2, e).
} 
protección de los Estados en desarrollo; recomendación, adopción y aplicación provisional de normas, reglamentos y procedimientos y aprobación de enmiendas a la Parte XI). ${ }^{14}$ Cuando entre en vigencia el Acuerdo debe intentarse lograr el consenso en relación con todas las decisiones que adopten todos los órganos de la Autoridad, inclusive cuando se trata de decisiones sobre cuestiones de procedimiento (el par. 2 de la Sección 3 no establece ninguna excepción), lo que ciertamente es una exigencia excesiva.

El requerir que se intente obtener un consenso antes de que una cuestión sea sometida a votación en los órganos principales de la Autoridad es una ilustración de la tendencia a recurrir a este método, que se ha venido manifestando últimamente de manera creciente en las conferencias y organizaciones internacionales, particularmente cuando se trata de adoptar decisiones y actos que tienen naturaleza normativa. El consenso ofrece ventajas obvias: asegura que no se tomarán decisiones que no reflejen un grado de aceptación general suficiente como para garantizar que ellas serán aplicadas efectivamente; al no exigir una manifestación de voluntad explícita, permite unirse al consenso a aquellos Estados que no apoyan totalmente la decisión pero que a la vez no desean aparecer impidiendo su adopción. Pero el método del consenso no está libre de fallas: para obtenerlo es a veces necesario embarcarse en interminables negociaciones que producen dilaciones y que frecuentemente terminan con la adopción de textos imprecisos o superfluos, o lo que es peor, con la paralización del órgano.

Sin embargo, en el sistema del Acuerdo el consenso no es el método final para la adopción de las decisiones en todos los casos, sino una etapa previa y obligada que debe intentarse antes de someter la cuestión a votación. Según lo dispone el par. 3 de la Sección 5 este método es mantenido como método final sólo en los asuntos para cuya decisión la Convención requiere el consenso del Consejo, a los que nos hemos referido ut-supra. Ello significa que el peligro de paralización o de terminar adoptando decisiones ambiguas o inefectivas se presentará precisamente cuando el Consejo desempeñe funciones normativas. Este problema resulta de las disposiciones de la Convención, no de las del Acuerdo [art. 162, par. 2, o)]. El consenso aparece como método único, además, para adoptar decisiones sobre cuestiones de fondo en el Comité

\footnotetext{
${ }^{14}$ Por otras decisiones del Consejo que deben ser adoptadas por consenso, ver Paolillo, op. cit., p. 236.
} 
de Finanzas, creado por la Sección 9 del Acuerdo (ver infra), lo cual puede conducir a similares resultados negativos en este órgano.

ii) El sistema de adopción de decisiones en la Ásamblea: Los párrafos 3 y 5 de la Sección 3 establecen el procedimiento de adopción de decisiones para la Asamblea y el Consejo respectivamente, en el caso de que hayan fracasado las tentativas para lograr el consenso. El Acuerdo mantiene el sistema establecido en la Convención para la adopción de decisiones en la Asamblea, disponiendo que una mayoría simple de miembros presentes y votantes es suficiente para adoptar una decisión sobre tina cuestión de procedimiento y que para adoptar una decisión sobre una cuestión de fondo se requiere una mayoría de dos tercios de Estados presentes y votantes. El Acuerdo no se refiere a los procedimientos especiales de los párrafos 9 y 10 del mismo artículo, pero puesto que no existen en el Acuerdo disposiciones incompatibles con ellos ni se declara la no aplicabilidad de los mismos, debe concluirse que se mantienen.

iii) El sistema de adopción de decisiones en el Consejo: Los cambios que introduce el Acuerdo en los procedimientos del Consejo para adoptar decisiones son radicales. Ante todo, reemplaza la barroca clasificación de cuestiones contenida en el art. 161, par. 8 de la Convención por la clásica distinción entre cuestiones de procedimiento y cuestiones de fondo. De este modo elimina támbién la panoplia de mayorías que la Convención prescribe para adoptar decisiones en cada una de las diferentes categorías de cuestiones.

Según el Acuerdo, fracasados los esfuerzos para obtener el consenso en el Consejo, las cuestiones de procedimiento son decididas con el voto de la mayoria de sus miembros presentes y votantes. Las decisiones sobre cuestiones de fondo serán tomadas por la mayoria de dos tercios de los miembros del Consejo presentes y votantes "a menos que se oponga a tales decisiones la mayoría de cualquiera de las cámaras mencionadas en el párrafo $9^{\prime \prime}$.

Las "cámaras" del Consejo no son en todos los casos los grupos de países establecidos en el párrafo 15 a los efectos de la composición del Consejo. Sólo los grupos descritos en los incisos a) (Estados grandes consumidores o importadores), b) (Estados mayores inversionistas en las actividades en la Zona), y c) (Estados grandes exportadores) constituyen cámaras en el sentido del pár. 9. En lo que respecta a los dos grupos restantes [Estados en desarrollo que representan intereses especiales (inciso d) y Estados elegidos para asegurar la representación geográfica equitativa en el Consejo en su totalidad (inciso e)], los paises en desarro- 


\section{F. H. Paolillo / Cuestiones institucionales en el ...}

1lo de ambos grupos constituyen una sola cámara a los efectos de la votación en el Consejo.

Ello quiere decir que para que una decisión sobre una cuestión de fondo pueda ser adoptada por el Consejo, será necesario el voto de los dos tercios de los miembros presentes y votantes que incluya a la mayoría de cada una de las cuatro cámaras. Como en el Acuerdo se ha eliminado el requisito de que la mayoría de los dos tercios debe incluir una mayoría de los miembros del Consejo como lo exigía el art. 161, par. 8, incisos b) y c) de la Convención, y como la mayoría de los miembros del Consejo constituyen quórum (art. 161, par. 6), la cantidad mínima de votos necesarios para adoptar decisiones sobre cuestiones de fondo oscilará entre 12 y 24 , según el Consejo esté funcionando con el quórum mínimo o con la totalidad de sus miembros.

Si la mayoría simple de cualquiera de las cuatro cámaras se opone a la decisión, ésta no puede ser adoptada cualquiera sea el número total de votos afirmativos que obtenga en el Consejo en su conjunto. Por medio de este mecanismo se otorga a los países que integran las tres cámaras que corresponden a los grupos de los incisos a), b) y c), -la mayor parte de los cuales serán países industrializados - un amplio poder para bloquear decisiones sobre cuestiones de fondo. Basta que tres paises que integran cualquiera de dichas cámaras se oponga a una decisión para que la misma no pueda ser adoptada, aun cuando los restantes 33 miembros del Consejo estén a favor de la misma. En cambio, los países en desarrollo necesitarán un número considerablemente mayor para lograr el mismo efecto. Como la cuarta cámara se integra con los países en desarrollo del grupo del inciso d) ( 6 miembros) más los países en desarrollo del grupo del inciso e) (probablemente no menos de 15 teniendo en cuenta que en la distribución geográfica equitativa de los puestos del Consejo en su totalidad le corresponderían a los países en desarrollo alrededor de 24 puestos), resulta que se requerirían en esta cámara por lo menos 11 votos para impedir que una decisión se adopte.

La aprobación de los planes de trabajo sigue un procedimiento especial. Es innecesario subrayar la importancia de esta cuestión: los planes de trabajo constituyen la herramienta legal que abre la Zona y sus recursos a los operadores que se proponen conducir actividades de exploración y explotación. El procedimiento que debía seguir una solicitud de aprobación de un plan de trabajo fue un punto extensamente debatido en la Conferencia entre los partidarios de otorgar a los órganos 
de la Autoridad amplia discrecionalidad para aprobar o no un plan de trabajo y aquéllos que querían dotar al procedimiento de la mayor automaticidad a fin de asegurar a los solicitantes un acceso sin obstáculos a la Zona y sus recursos. El procedimiento que finaimente se incorporó en la Convención [art. 162, par. 2,j)] garantiza un acceso casi automático a aquellos solicitantes que hubieran llenado ciertos requisitos previamente establecidos, eliminando de este modo la posibilidad de que factores políticos $u$ otros factores circunstanciales sudieran influir en los órganos encargados de tomar las decisiones.

El Acuerdo dispone la inaplicabili lad del apartado j) del párrafo 2 del art. 162 [Sección 3, pár. 11, b)]. Si bien mantiene el principio de que las decisiones de aprobar o no aprobar los planes de trabajo son adoptadas por el Consejo sobre la base de una recomendación de la Comisión Jurídica y Técnica, cambia los otros aspectos del procedimiento. Si la Comisión Jurídica y Técnica ha recomendado aprobar un plan de trabajo, será necesaria una mayoría de dos tercios de miembros del Consejo presentes y votantes que incluya la mayoría de miembros presentes y votantes de cada una de sus cámaras para no aprobar el plan de trabajo. Si tal mayoría no se opone o si el Consejo no se expide dentro de un plazo prescripto (que normalmente será de 60 dias) se considera que el plan de trabajo queda aprobado. De este modo se hace verdaderamente dificil que un plan de trabajo recomendado favorablemente por la Comisión pueda ser rechazado por el Consejo. Si la recomendación de la Comisión es negativa, o si ella no formula recomendación alguna, el Consejo de todos modos podrá aprobar el plan de trabajo, pero para ello deberá reunir la mayoría requerida para decidir cuestiones de fondo [Sección 3, pár. $11, \mathrm{a})]$.

Es claro que el sistema de votación de los planes de trabajo ha sido despojado de los complicados trámites prescriptos en el apartado j) del pár. 2 del art. 162. El procedimiento ahora es más automático y la adopción de una decisión toma menos tiempo. El pár. 12 de la Sección 3 agrega que las controversias que se produjeran con respecto al rechazo de un plan de trabajo serán sometidas al procedimiento de solución đe controversias establecido en la Convención, es decir, a la Sala de Controversias de los Fondos Marinos [art. 187, apartado d)].

Al estar basado en la elemental clasificación de cuestiones de fondo y cuestiones de procedimiento y no en la enumeración casuística de cuestiones como lo hace la Convención, el sistema de votación del 


\section{F. H. Paolillo / Cuestiones institucionales en el ...}

Consejo, tal como lo propone el Acuerdo, es considerablemente más simple, elimina la posibilidad de vacíos legales y evita incongruencias tales como las de exigir mayorías especiales para decidir cuestiones cuyo carácter procesal o cuya importancia secundaria está fuera de toda duda. $^{15}$

\section{VI}

\section{Cambios en las normas de integración del Consejo.}

El Acuerdo introdujo importantes cambios en la forma de integrar el Consejo, tema que está íntimamente vinculado al procedimiento de adopción de decisiones en dicho órgano. La integración del Consejo fue otra de las cuestiones que demandó una larga discusión en la Conferencia. En tanto que el Grupo de los 77 proponía que el principio básico de integración del Consejo fuera el de la representación geográfica equitativa, los países industrializados insistieron en la representación de los intereses especiales de los Estados que conducirían actividades de explotación en la Zona. El resultado fue, también en este tema, un sistema complicado en el que se dio cabida tanto al principio de la representación geográfica [art. 161, par. 1, e)] como al de la representación de intereses económicos [(art. 161, par. 1, a), b) y c)], así como también al de la representación de otros intereses "especiales" que incluye a los Estados con gran población, los Estados sin litoral o en situación geográficamente desventajosa y los Estados en desarrollo menos adelantados [art. 161, par. 1, d)].

El Acuerdo dispone la inaplicabilidad de ]as disposiciones del párrafo 1 del artículo 161 de la Convención sobre la composición del Consejo (Sección 3, par. 16), pero en el pár. 15 de la misma Sección mantiene la estructura general de dicha disposición con la categorización de los miembros del Consejo en cinco grupos. Aparte de algunas enmiendas de carácter formal, (en el inc. e) se agrega una referencia al Caribe al designar a la región de América Latina; se suprime la palabra "socialista" al designar al grupo de Europa Oriental). Los cambios introducidos tienen por fin asegurar una identificación más precisa de los países que

\footnotetext{
${ }^{15}$ Un ejemplo: la creación de órganos subsidiarios debe ser resuelta, según el sistema de la Convención, por una mayoria de tres cuartos de los miembros del Consejo (art. 161, par. 8, c).
} 
representarán en el Consejo al primer grupo de interés [mayores consumidores e importadores de productos básicos derivadós de las categorías de minerales a extraerse de la Zona, inc: a)].

En el sistema consagrado en la Convención, la descripción de los intereses representados en las tres primeras categorías [incisos a), b) y c)], aseguraba al grupo (relativamente pequeño) de los países industrializados con perspectivas de realizar actividades en la Zona la presencia casi permanente en el Consejo de algunos de sus miembros gracias a sucesivas elecciones o reelecciones, en la misma o en diferentes categorías. Debe recordarse, además, que los países industrializados pueden acceder al Consejo no sólo como integrantes de las tres primeras categorías, sino también como representantes del grupo geográfico de Europa Occidental y otros Estados en la categoría descrita en el inciso d).

Aun así, algunos Estados entendieron que el sistema de la Convención no les ofrecía suficientes garantías de una representación permanente en el Consejo, a lo cual aspiraban, en especial los Estados Unidos. El Acuerdo, entonces, modifica el inciso a) agregando que entre los cuatro Estados elegidos dentro de esta categoría (Estados Partes que, durante los últimos cinco años hayan absorbido más del $2 \%$ en términos de valor del consumo mundial o hayan importado mas del $2 \%$ en términos de valor de las importaciones mundiales totales de productos básicos obtenidos de minerales de la Zona) debe incluirse

"... a un Estado de la región de Europa oriental que tenga la economía más importante de esa región en términos de producto intemo bruto, y al Estado que a la fecha de entrada en vigor de la Convención, tenga la economía más importante en términos de producto interno bruto, si esos Estados desean estar representados en este Grupo".

Esos dos asientos en el Consejo tienen, por supuesto, nombre propio. Pero en tanto que el que le corresponde a los Estados Unidos ha de ser ocupado en el futuro exclusivamente por dicho país, puesto que el requisito exigiđo se cumplió una vez y para siempre al entrar en vigor la Convención, ${ }^{16}$ el que ahora corresponde a Rusia por tener la economía más importante de la región podría ser ocupado en el futuro por otro país

\footnotetext{
${ }^{16}$ No se entiende por qué se le da a los Estados Unidos la opción đe "no estar representado en este grupo". En el hipotético caso de que los Estados Unidos desearan ser elegidos en otro grupo, y puesto que sólo este país llena la condición de ser el país con la economía más importante a la fecha de entrada en vigor de la Convención, ¿queda este asiento del primer grupo sin llenar?
} 
de la misma región si las condiciores económicas cambian substancialmente.

Dos alteraciones más se introdujeron en la composición del Consejo: en primer lugar, se eliminó el requisito de que entre los miembros del segundo grupo (inc. b: Estados Partes que hayan hecho las mayores inversiones en la preparación y realización de actividades en la Zona) haya por lo menos un Estado de la región de Europa Oriental; y, en segundo lugar, se agregó el interés de los Estados insulares en desarrollo a la lista de los intereses especiales (inc. d).

\section{VII}

\section{El Comité de Finanzas.}

El Acuerdo contiene otras disposiciones relativas a la estructura y funcionamiento de la Autoridad que tienen incidencia en el sistema de adopción de decisiones de los órganos principales. Las más importantes son, tal vez, aquéllas por las que se crea el Comité de Finanzas, contenidas en la Sección 9 del Acuerdo.

La Convención, que establece dos órganos subsidiarios del Consejo (la Comisión de Planificación Económica y la Comisión Jurídica y Técnica), cuya organización, competencia y funcionamiento son objeto de los arts. 163 a 165, no estableció ningún órgano subsidiario para entender en las cuestiones financieras de la Autoridad, pero previó su creación, también como órgano subsidiario del Consejo [art. 162, par. 2, y)].

De conformidad con el Acuerdo, al entrar en vigor la Convención entrarán en funcionamiento, además de los órganos principales de la Autoridad, la Comisión Jurídica y Técnica y el Comité de Finanzas. Las funciones de la Comisión de Planificación Económica creada por la Convención serán desempeñadas por la Comisión Jurídica y Técnica hasta tanto el Consejo decida otra cosa o hasta que se apruebe el primer plan de trabajo para la explotación de recursos (Sección 1, par. 4).

Con la creación del Comité de Finanzas, el Acuerdo entiende cumplir con el mandato de la Convención contenido en el art. 162, par. 2, y) que dispone el establecimiento de un órgano, que funcionará como órgano subsidiario del Consejo, encargado de la elaboración de los proyectos de normas, reglamentos y procedimientos financieros de la Autoridad. Sin 
embargo, no resulta claro del Acuerdo si el Comité de Finanzas ha sido creado como órgano subsidiario del Consejo. Curiosamente los miembros de este Comité son elegidos por la Asamblea (Sección 9, par. 3), contrariamente a lo que la Convención dispone con respecto a la Comisión de Planificación Económica y la Comisión Jurídica y Técnica, cuyos miembros son elegidos por el Consejo sin que la Asamblea tenga ninguna participación en ello.

En la elección de los 15 miembros del Comité de Finanzas, la Asamblea deberá tener en cuenta la necesidad de una distribución geográfica equitativa y la representación de intereses especiales. Los candidatos que presenten los Estados Partes deberán ser personas "de competencia e integridad máximas" (Sección 9, párs. 1 y 3).

El par. 3 de la Sección 9 dispone que el Comité de Finanzas estará integrado por lo menos por un miembro que represente cada uno de los grupos de Estados a los que se refieren los apartados a), b), c), y d) del par. 15 de la Sección 3. Además, y hasta tanto la Autoridad adquiera autonomía financiera para sufragar sus gastos administrativos, representantes de los cinco mayores contribuyentes financieros al presupuesto de la Autoridad deberán formar parte del Comité.

Otro rasgo del Comité de Finanzas que llama la atención es el de que las decisiones sobre cuestiones de fondo deberán ser adoptadas por consenso [Sección 9, pár. 8). Parece un poco excesivo exigir consenso en este órgano subsidiario que posee solamente poderes de recomendación. Las decisiones finales sobre asuntos financieros serán tomadas en la Asamblea o/y el Consejo, en donde otra vez se requerirá intentar la obtención de un consenso como etapa previa al acto de votación. En contraste, en la Comisión Jurídica y Técnica, el otro órgano subsidiario cuyo funcionamiento está previsto en el Acuerdo, las decisiones podrán ser adoptadas por mayoría de los miembros presentes y votantes (Sección 3, pár. 13), luego, naturalmente, de haberse intentado el consenso como lo requiere el pár. 2 de la misma Sección para todos los órganos de la Autoridad. Considerando la amplia competencia que la Convención le acordó a la Comisión Técnica Jurídica (art. 165), el método de adopción de decisiones de esta Comisión aparece considerablemente menos exigente comparado con el que deberá aplicar el Comité de Finanzas.

El Comité queda encargado de examinar una serie de cuestiones de carácter financiero que se enumeran en el par. 7 de la Sección 3, y de formular recomendaciones sobre ellas a la Asamblea y al Consejo. Las decisiones de los dos órganos. principales respecto de estas cuestiones deberán adoptarse teniendo en cuenta las recomendaciones del Comité. 
No se especifica en el Acuerdo el modo cómo intervienen la Asamblea y el Consejo en la consideración y resolución de estas cuestiones. En todo caso, dado que se trata de asuntos con respecto a los cuales la competencia es compartida por los dos órganos principales, la Asamblea deberá basar sus decisiones en las recomendaciones del Consejo, tal como lo prescribe el párrafo 4 de la Sección 3.

\section{VIII}

Las disposiciones del Acuerdo sobre aspectos institucionales de la $\mathrm{Au}-$ toridad introducen cambios profundos en su funcionamiento tal como lo preveía la Convención, que afectan no solamente a los procedimientos para adoptar decisiones, sino además a la distribución de competencias entre los órganos principales y a la composición del Consejo. El resultado final es el de asegurar una mayor influencia de los países industrializados en las decisiones relativas a la administración de la Zona. Ello se ha logrado alterando la distribución de competencias entre los dos órganos principales de la Autoridad en beneficio del Consejo y modificando los procedimientos para adoptar decisiones.

La expansión de la competencia del Consejo implica, naturalmente, un debilitamiento de la Asamblea, órgano plenario de la Autoridad. La pérdida, en beneficio del Consejo, del poder de adoptar de manera autónoma la política de la Autoridad no hace más que acentuar el carácter supremo que el Consejo ya poseía en el sistema de la Convención. La supremacía de la Asamblea no era más que aparente en la Convención; después del Acuerdo perdió incluso el viso de apariencia. Los cambios en los procedimientos de votación en el Consejo colocan a los paises industrializados en posición de poder orientar la gestión de la Autoridả en la dirección que mejor satisfaga a sus intereses. Por otro lado, al simplificar los procedimientos de votación en el Consejo, el Acuerdo mejora considerablemente el complicado sistema establecido en la Convención.

El sistema establecido por el Acuerdo cumple con el objetivo perseguido por los países industrializados, en particular aquellos que proyectan emprender actividades de explotación de los recursos de la Zona, de fortalecer su posición dentro del Consejo, y de fortalecer la posición del Consejo dentro de la Autoridad. De este modo han aumentado, sin duda, las perspectivas de lograr, por fin, la vigencia general de la Convención -pero de una Convención modificada-. 